\title{
Colonic Lymphangiomatosis Resolved after Excisional Biopsy
}

\author{
Young Soo Lee', Gyu Won Kim¹, Hye Jae Cho and Chan Sup Shim ${ }^{3}$ \\ Departments of ${ }^{1}$ Internal Medicine and ${ }^{2}$ Pathology, Sahmyook Medical Center, Seoul, ${ }^{3}$ Division of Gastroenterology, Department of Internal Medicine, \\ Konkuk University Medical Center, Konkuk University School of Medicine, Seoul, Korea
}

Lymphangioma is an uncommon malformation of the lymphatic system that involves a benign proliferation of the lymphatics, with no established treatment method. Multiple colonic lymphangioma, or colonic lymphangiomatosis, is an extremely rare condition. We report a case of colonic lymphangiomatosis that was detected during a colonoscopic examination conducted as part of a general health checkup. The lesion completely resolved after excisional biopsy.

Key Words: Colon; Excision; Lymphangioma; Lymphangiomatosis; Resolution

\section{INTRODUCTION}

Lymphangioma is a developmental malformation of lymphatic tissue, characterized by submucosal tumors covered with normal mucosa. ${ }^{1}$ These benign tumors are commonly found in children, and although they may develop anywhere, the tumors are usually found in the head, neck, and axillary regions, and rarely in the gastrointestinal tract. ${ }^{2}$ Most reported cases of lymphangioma of the colon are solitary; few cases involve multiple lymphangioma, also known as colonic lymphangiomatosis. ${ }^{1}$ Colonic lymphangiomas are typically asymptomatic, are discovered only incidentally, and usually do not require treatment..$^{3-5}$ However, in some cases, they may cause abdominal pain, bleeding, intussusception, or protein-losing enteropathy, requiring surgical or endoscopic resection. ${ }^{1,6}$

\section{CASE REPORT}

A 38-year-old man visited our comprehensive medical center for a general check-up. He had no previous medical problems other than diabetes mellitus and essential hypertension,

Received: January 5, 2014 Revised: April 2, 2014

Accepted: May 12, 2014

Correspondence: Chan Sup Shim

Division of Gastroenterology, Department of Internal Medicine, Konkuk University Medical Center, Konkuk University School of Medicine, 120 Neungdong-ro, Gwangin-gu, Seoul 143-729, Korea

Tel: +82-2-2030-5026, Fax: +82-2-2030-5029, E-mail: chansshim@naver.com

(c) This is an Open Access article distributed under the terms of the Creative Commons Attribution Non-Commercial License (http://creativecommons.org/ licenses/by-nc/3.0) which permits unrestricted non-commercial use, distribution, and reproduction in any medium, provided the original work is properly cited. which were diagnosed 1 year prior at another hospital. He was $176 \mathrm{~cm}$ tall and weighed $104 \mathrm{~kg}$. Laboratory test results, including the levels of tumor markers carcinoembryonic antigen and carbohydrate antigen, were all within normal ranges, except the levels of triglycerides at $206 \mathrm{mg} / \mathrm{dL}$ (normal, 10 to 180 ) and hemoglobin A1c at 9.3\% (normal, 4.0 to 6.0). Electrocardiography and chest and abdominal radiography did not reveal any abnormal findings. Esophagogastroduodenoscopy showed hyperemic mucosal change in the antrum and body of the stomach, with multiple shallow erosions in the second portion of the duodenum. An initial colonoscopy revealed multiple, variably sized cystic mass lesions in the midportion of the ascending colon up to the proximal portion of the transverse colon (Fig. 1). Two biopsy specimens were taken from those tumors. Abdominal ultrasonography and computed tomography (CT) revealed no abnormalities other than a fatty liver (Fig. 2). Histopathological examination showed chronic inflammation, with no prominent cystic change.

The patient underwent a follow-up colonoscopy 6 weeks after the first colonoscopy. The cystic lesion on previous colonoscopy was again visualized. We decided to perform an excisional biopsy to accurately diagnose the lesion. A solution composed of hypertonic saline, epinephrine, and methylene blue was injected around some of the cystic masses and biopsy specimens were taken with snare electrocautery (Fig. 3). During the procedure, incision on one of the masses using a dual knife caused clear yellow fluid to flow out from the mass, after which the mass began to shrink. The biopsied tissue revealed normal colonic mucosa and markedly dilated lymphatic ves- 


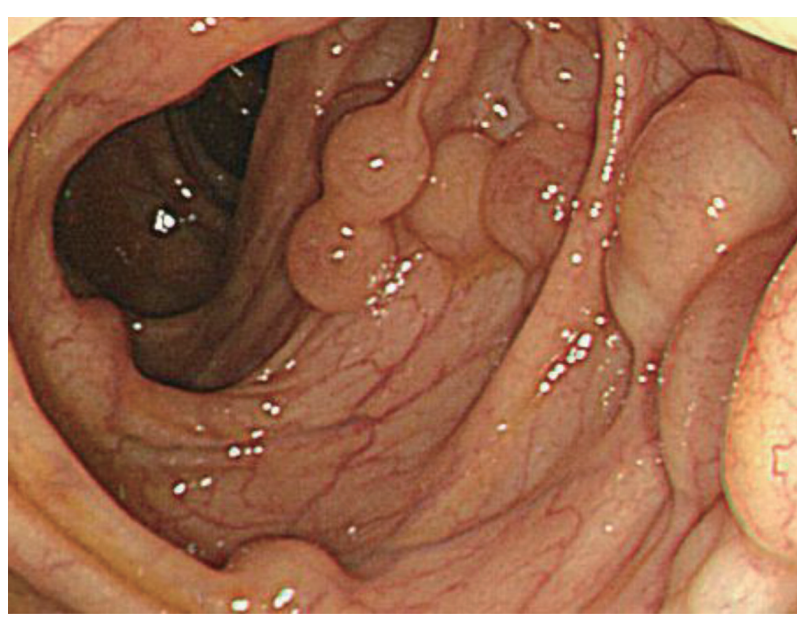

Fig. 1. Colonoscopy revealing a cluster of multiple cystic masses of various sizes and shapes with the same color and surface as the surrounding normal mucosa.

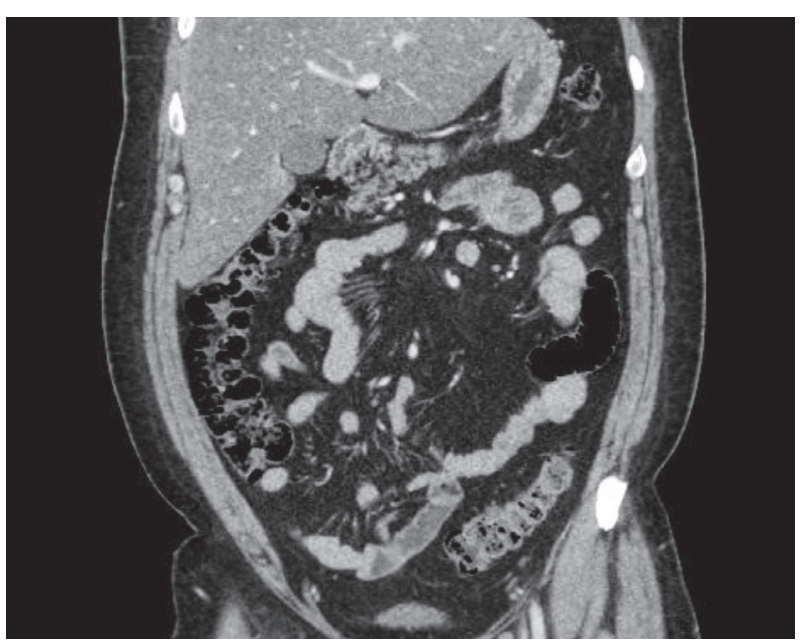

Fig. 2. Abdominal computed tomography scan showing normal colonic findings.

sels in the submucosa. Immunohistochemical staining for CD34 and D2-40 showed a positive reaction along the lining cells of the dilated lymphatic channels (Fig. 4). CD34 is an endothelial cell marker in different vascular beds in human tissues, ${ }^{7}$ and the monoclonal antibody D2-40 is a marker of lymphatic endothelium. ${ }^{8}$ D2-40 does not stain vascular endothelium.

Eight months later, the patient underwent a third colonoscopic examination that showed normal colonic mucosa without the protruded lymphangiomatosis previously been observed in the ascending and transverse colon. No scarring or trace of the former lesions was found in the entire colon (Fig. 5).

\section{DISCUSSION}

Lymphangioma is regarded as a developmental malformation of lymphatic tissue; obstruction or agenesis of lymphatic tissue causes abnormal dilatation and proliferation of the lymphatic channel, leading to the formation of a cystic mass. ${ }^{3,4} \mathrm{Se}-$ condary lymphangioma formation can be caused by abdominal trauma, lymphatic obstruction, inflammatory processes, cancer, surgery, or radiation. In the present case, colonic lymphangiomatosis was discovered in adulthood, suggesting that the lesion developed secondary to a local disturbance of the lymphatic circulation. However, the patient had no specific history related to colonic lymphangiomatosis, and abdominal CT showed no comorbid disease; thus, it would be more rational to conclude that lymphangioma developed primarily in the fetal period in this case, and was silent until detection on medical examination.

Lymphangiomas rarely occur in the abdomen, and the mesentery, omentum, mesocolon, and retroperitoneum are the most common sites. ${ }^{9}$ The incidence of lymphangioma in the intestinal wall is very low, and few cases of multiple colonic lymphangioma have been reported. ${ }^{1}$ In the present case, multiple lymphangiomas of the colon, known as colonic lymphangiomatosis, were observed, and had resolved by the time of the third colonoscopic examination.

The natural history of lymphangioma has not been clearly defined. It is considered benign; no cases of malignant transformation have been reported. ${ }^{1}$ Trials have been conducted to explain how lymphangiomas may be resolved. Several investigators have suggested that increased pressure in the lymphatic system may overcome incomplete obstructions. Others have hypothesized that resolution may occur through the establishment of alternative routes of lymphatic drainage. ${ }^{10}$ The latter theory seems to fit well with the present case. It is possible that in the course of making incisions and taking excisional biopsies of the colonic lymphangiomatosis, conduits that drain lymphatic outflow toward the colonic lumen were formed, leading to the complete resolution of the lymphangiomatosis.

There is no standard approach to the treatment of intra-abdominal or intraluminal lymphangiomas. Historically, both were treated by surgical resection due to uncertainty regarding the nature of the lesion. However, since lymphangioma is a benign tumor, and most intra-abdominal or intestinal lymphangiomas are asymptomatic, they usually do not require treatment. ${ }^{3-5}$ Kochman et al. ${ }^{5}$ suggested that asymptomatic lymphangiomas should be observed rather than treated. Large lymphangiomas may produce bowel obstruction, intussusception, bleeding, anemia, and protein-losing enteropathy, in addition to symptoms such as abdominal pain, vomiting, and diarrhea. ${ }^{6}$ Complete surgical excision is considered the treatment of choice for these large, symptomatic lymphangiomas. ${ }^{1,6}$ For pedunculated tumors $<2 \mathrm{~cm}$, recent reports indicate a trend towards the use of endoscopic polypectomy. ${ }^{11}$ In the 

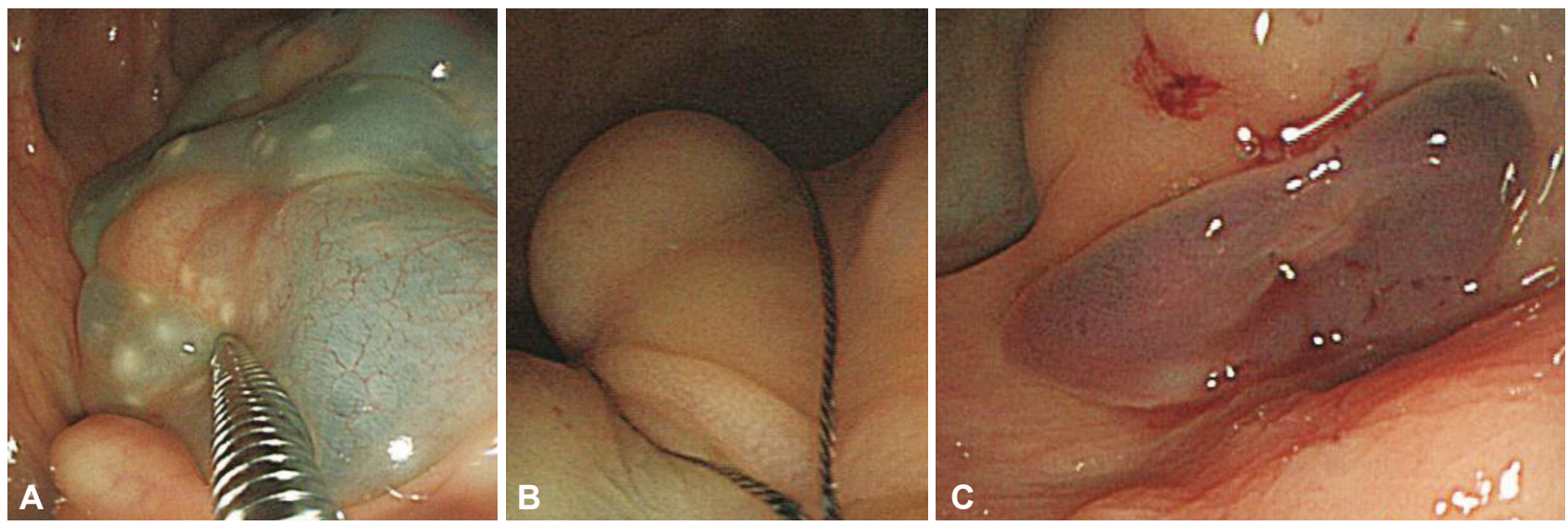

Fig. 3. (A) Lifting solution is injected around the cystic masses. (B) A snare is applied to the injected cystic mass. (C) Shrunken mass after incision.
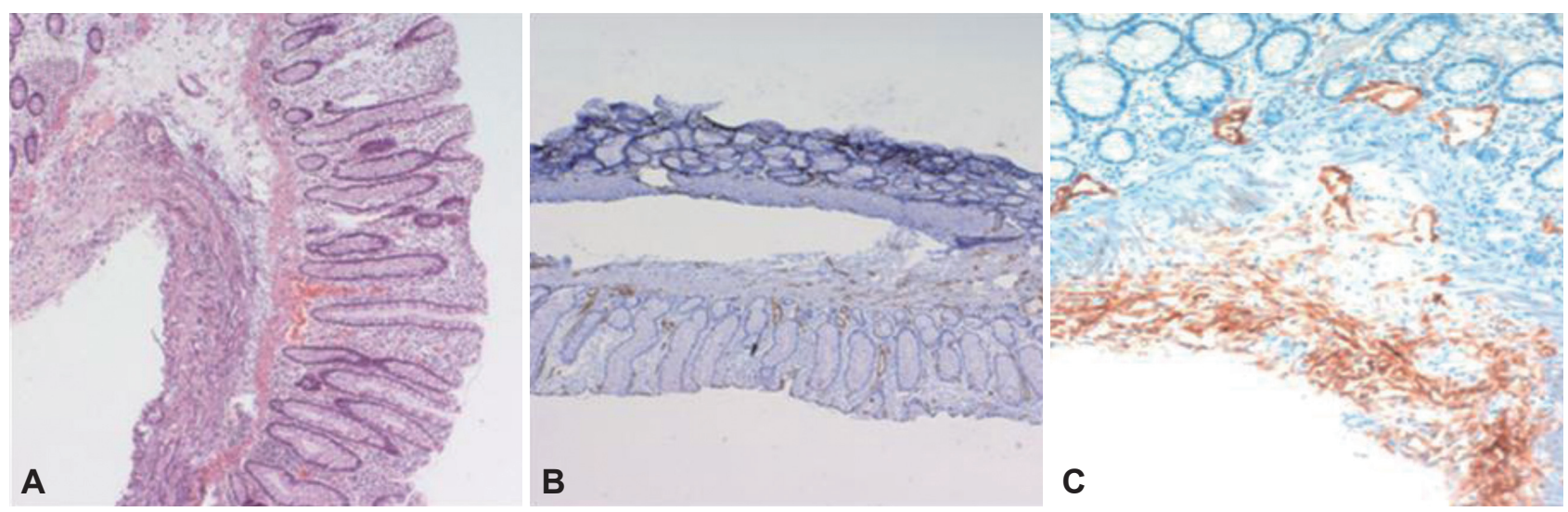

Fig. 4. (A) Hematoxylin and eosin staining of normal colonic mucosa and a markedly dilated lymphatic vessel $(\times 100)$. (B) CD34 expression in a lymphatic vessel. Smooth muscle cells and endothelial cells are located beneath the colonic mucosal glands $(\times 100)$. (C) D2-40 expression (brown color) in a lymphatic vessel $(\times 200)$.

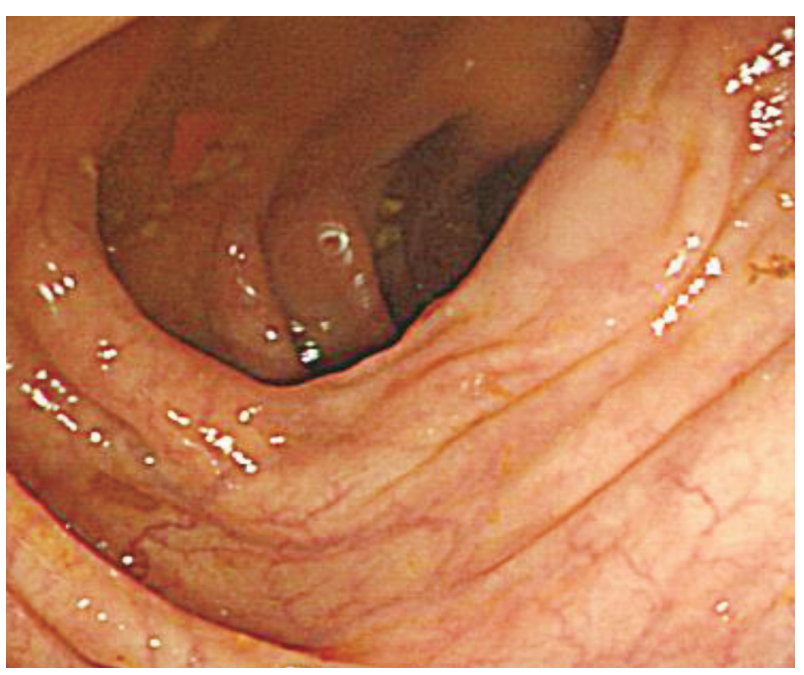

Fig. 5. Normal colonic mucosa is seen on third colonoscopy. No abnormality including previous lymphangiomatosis is found.

present case, no treatment other than several incisions and excisional biopsies was attempted because the observed tumors were all small, sessile, and did not exhibit symptoms or cause complications. In a follow-up study after the incisions and excisional biopsies, we found that such tumors were completely resolved. Our assumption is that conduits were made at some sites of incisions or excisions, and lymphatic drainage through those conduits from the lymphatic channel to the colonic lumen brought about the resolution of the colonic lymphangiomatosis. During 8 months of surveillance after the procedure, no complications such as bleeding or protein-losing enteropathy were noticed.

This is a case report of a patient in whom dozens of lymphangiomas were found in the ascending colon up to the transverse colon, which was observed and confirmed through colonoscopy and biopsy. The colonic lymphangiomatosis was observed to be completely resolved in follow-up studies, without any treatment.

\section{Conflicts of Interest}

The authors have no financial conflicts of interest.

\section{REFERENCES}

1. Watanabe T, Kato K, Sugitani M, et al. A case of multiple lymphangio- 
mas of the colon suggesting colonic lymphangiomatosis. Gastrointest Endosc 2000;52:781-784.

2. Pipinos II, Baxter BT. The lymphatics. In: Townsend CM, Beauchamp RD, Evers BM, et al., eds. Textbook of Surgery: the Biological Basis of Modern Surgical Practice. 17th ed. Philadelphia: W.B. Saunders; 2004. p. 2078.

3. Jung SW, Cha JM, Lee JI, et al. A case report with lymphangiomatosis of the colon. J Korean Med Sci 2010;25:155-158.

4. Lee JM, Chung WC, Lee KM, et al. Spontaneous resolution of multiple lymphangiomas of the colon: a case report. World J Gastroenterol 2011;17:1515-1518.

5. Kochman ML, Wiersema MJ, Hawes RH, Canal D, Wiersema L. Preoperative diagnosis of cystic lymphangioma of the colon by endoscopic ultrasound. Gastrointest Endosc 1997;45:204-206.

6. Matsuda T, Matsutani T, Tsuchiya Y, et al. A clinical evaluation of lymphangioma of the large intestine: a case presentation of lymphan- gioma of the descending colon and a review of 279 Japanese cases. J Nippon Med Sch 2001;68:262-265.

7. Pusztaszeri MP, Seelentag W, Bosman FT. Immunohistochemical expression of endothelial markers CD31, CD34, von Willebrand factor, and Fli-1 in normal human tissues. J Histochem Cytochem 2006;54: 385-395.

8. Fukunaga M. Expression of D2-40 in lymphatic endothelium of normal tissues and in vascular tumours. Histopathology 2005;46:396-402.

9. Enzinger FM, Weiss SW. Tumors of lymph vessels. In: Enzinger FM, Weiss SW, eds. Soft Tissue Tumors. 3rd ed. St. Louis: Mosby; 1995. p. 679-699.

10. Bernstein HS, Filly RA, Goldberg JD, Golbus MS. Prognosis of fetuses with a cystic hygroma. Prenat Diagn 1991;11:349-355.

11. Sato K, Maekawa T, Yabuki K, et al. Cystic lymphangiomas of the colon. J Gastroenterol 1999;34:520-524. 